\title{
What have we learnt after 15 years of research into the 'weekend effect'?
}

\author{
Benjamin D Bray, ${ }^{1}$ Adam Steventon ${ }^{2}$
}

${ }^{1}$ Farr Institute of Health Informatics Research, University College London, London UK ${ }^{2}$ Department of Data Analytics, The Health Foundation, London, UK

\section{Correspondence to} Dr Adam Steventon, Data Analytics, The Health Foundation, 90 Long Acre, London WC2E 9RA, UK ; adam.steventon@health.org.uk

Accepted 21 October 2016 Published Online First 30 November 2016

\section{SLinked}

- http://dx.doi.org/10.1136/ bmjqs-2016-005680

\section{CrossMark}

To cite: Bray $B D$, Steventon $A$. BMJ Qual Saf

2017;26:607-610.
It is now 15 years since Bell and Redelmeier published their landmark study demonstrating higher mortality for people admitted to hospital during weekends compared with during the week. ${ }^{1}$ Examining the records of 3.8 million patients admitted over a 10 -year period to emergency departments in Ontario, Canada, this 'weekend effect' existed over a range of acute conditions, including 23 out of the 100 leading causes of death.

Since that paper in 2001, over 100 studies have explored the weekend effect, across a range of patient populations and health systems. ${ }^{2}$ Surprisingly, despite this large number of studies, there remains ongoing debate about whether the weekend effect exists, and if so, what causes it. For example, one recent and highly influential study found higher rates of in-hospital death following admission on Saturday or Sunday compared with Wednesday admissions (HR 1.10 for Saturday and 1.15 for Sunday). ${ }^{3}$

Policy makers and politicians in England have frequently referred to this result and those of other research studies on the weekend effect in justifying existing moves to provide more consistent 7-day health services. ${ }^{4}$ The nature of some of the proposed measures, including changes in doctors' work contracts, have proven extremely controversial, contributing to the first large-scale strike by doctors in England in over 40 years. $^{5}$ Controversies over the weekend effect have not been limited to the political arena. Some researchers have questioned the existence of the weekend effect, ${ }^{6}$ while others have highlighted the uncertainty about what causes it. ${ }^{2}$ Given these persistent controversies and questions, including those generated by the paper by Anselmi et $a l^{7}$ in this issue, it seems fitting to ask what have we really learnt about the quality and safety of healthcare from 15 years of studies into the weekend effect.

\section{IS THE WEEKEND EFFECT REAL OR SIMPLY AN ARTEFACT OF THE DATA?}

One of the major issues in untangling the weekend effect has been in taking account of differences in the patients admitted during the week compared with the weekend. For some conditions at least, the characteristics of patients attending hospital at the weekend differ from weekdays, with patients at the weekend having a higher average illness severity. ${ }^{8}$

Many studies of weekend effects have used administrative data, which were originally collected for the purposes of the day-to-day management of healthcare services (eg, for the reimbursement of services provided by hospitals), rather than research. ${ }^{9}$ One of their attractions is that, since administrative data are collected for all admissions, studies using these data are not limited to certain conditions or a sample of patients. Administrative data can be used to take account of some of the most important predictors of mortality (including age, comorbidity and the route of admission), but they have limitations. For example, in a study using administrative data, a 'pneumonia' is a 'pneumonia', irrespective of whether it is a clinically minor lower respiratory tract infection or a more life-threatening infection. In some other contexts, there is quite strong evidence that analysis can be biased when using administrative data for case-mix adjustment, ${ }^{10}$ but the implications for the weekend effect are unclear. The lack of clinical detail might bias the estimated weekend effect more heavily for some conditions than others, ${ }^{11}$ while for certain conditions the accuracy of coding has also been shown to affect these estimates. ${ }^{12}$ 
Meacock et $a l^{13}$ examined emergency departments in England between April 2013 and February 2014 and compared the evidence for a weekend effect among patients who attended an emergency department $(n=12670788)$ and the subset of patients $(29 \%, n=3715727)$ who were subsequently admitted for inpatient care. They found no evidence for a weekend effect among the broader set of patients attending emergency departments (risk-adjusted OR $1.010,95 \%$ CI 0.997 to 1.022 ). However, the admission rate from emergency departments was lower during the weekend than the week $(27.5 \%$ vs $30.0 \%)$ and, among the subset of patients who were admitted through that route, there was higher mortality among those admitted at the weekend (adjusted OR, 1.054, $95 \%$ CI 1.040 to 1.069$)$. This suggests that differences in admission practices might be one explanation for the apparently higher mortality risk at the weekend.

Building on this earlier work, the same group produced the paper by Anselmi et al in this issue, further developing the risk-adjustment method for the subset of patients admitted to hospital through an emergency department. ${ }^{7}$ By linking the emergency department and inpatient data, these authors were able to control for whether or not the patient arrived by ambulance, in addition to variables adjusted for in previous studies. As might be expected, patients who arrived at an emergency department by ambulance had a much higher mortality rate than those who arrived by other modes (crude mortality rate $5.46 \%$ vs $0.78 \%$ ), and the proportion arriving by ambulance was slightly higher at the weekend than during the week $(63.6 \%$ of patients vs $60.8 \%)$. When additionally controlling for arrival mode, the weekend effect almost halved compared with conventional risk adjustment (adjusted OR of 1.024 vs 1.046). That the estimates were so sensitive to the inclusion of one additional variable in the risk adjustment model illustrates the nebulous and contentious nature of the weekend effect. What is unknown is whether the remaining difference in mortality rates would persist with additional data on acute disease severity.

Some studies have access to richer clinical data that contain more information about illness severity. For example, one study adjusted for a standardised measure of acuity known as the National Early Warning Score. ${ }^{8}$ After adjusting for this, the weekend effect did not remain in that study, but due to a limited sample size the authors were not able to exclude the possibility of a small but potentially real difference in mortality. Still other studies have adjusted for condition-specific measures of severity, although the overall pattern in relation to the weekend effect remains mixed. ${ }^{14} 15$

Overall, although many studies find evidence of higher mortality rates for patients admitted at the weekend, it remains unclear to what extent this weekend effect reflects real differences in the quality of care versus methodological subtleties or data artefacts. If nothing else, these studies have served the useful purpose of highlighting some of the pitfalls and complexities involved in analysing administrative data. Administrative data are potentially very valuable resources for research and improvement efforts, ${ }^{9}$ but may not have the level of detail required to untangle complex issues such as the weekend effect.

\section{IF THE WEEKEND EFFECT IS REAL, WHAT CAUSES IT?}

Moving from merely observing the weekend effect to doing something meaningful to mitigate or prevent it requires a good understanding of why it occurs. Here unfortunately the body of research into the weekend effect thins out dramatically, with only a handful of studies addressing questions about causes and mechanisms.

One of the most commonly cited potential causes of the weekend effect relates to staffing at the weekend. Indeed, Bell and Redelemeier ${ }^{1}$ motivated their study with the observation that "staffing levels in acute care hospitals tend to be lower on weekends than on weekdays" and they went on to point out that, at least in Ontario circa 2001, clinicians who work on weekends often have less seniority and experience than those who work on weekdays, and may provide coverage for other health professionals and thus be less familiar with the patients under their charge. Although those authors suggested that staffing differences might explain their weekend effect, they did not test such a hypothesis formally.

The studies that have specifically addressed the role of staffing levels on the weekend effect suggest a more complicated story than a simple lack of doctors at the weekend. A recent large cross-sectional study of hospitals in England found that although staffing levels by specialist physicians were lower at the weekend relative to the number of emergency admissions, there was no evidence for a correlation between the ratio of weekend to weekday specialist staffing and the weekend effect. ${ }^{16}$ This is consistent with another study finding that there was no evidence that the higher mortality rates seen for surgical patients following weekend admission were explained by the level of experience of the operating surgeon. ${ }^{17}$ Similarly, a study of acute stroke care found no association between 7 day rounds by stroke specialist physicians and patients' risk of dying if admitted at the weekend. ${ }^{18}$ By contrast, in that study, low nurse staffing levels were strongly correlated with higher mortality rates subsequent to weekend admission. ${ }^{18}$ Although the evidence is still scanty, at present it seems unlikely that a relative lack of doctors working at the weekend entirely explains the weekend effect, and other organisational factors might be more important. 


\section{IS THERE A LINK BETWEEN THE WEEKEND EFFECT AND CARE PROCESSES?}

Studies have shown that other measures of care quality also vary across the week. For example, there is variation across rates of coronary intervention for patients with myocardial infarction, ${ }^{15} 19$ and rates of thrombolysis and treatment times for patients with acute stroke. ${ }^{1420}$ In addition to helping to explain differences in mortality, variation in evidence-based care processes represent potentially important inequalities in care quality in their own right, such as reduced access to evidence-based interventions.

Studies of care processes have helped to shed light on more complex patterns of variation in care quality beyond simple binary comparisons between the weekend and weekday. A detailed time-stratified analysis of stroke care found that the weekend effect was a simplification of much more complicated patterns of care quality that extended across the whole 24/ 7 week. ${ }^{14}$ While some aspects of care changed sequentially across the week, there were also diurnal variations in care quality. For example, the odds of being admitted to a stroke unit within 4 hours improved sequentially across weekdays before deteriorating at the weekend, with the lowest odds on Mondays. But at the same time, patients arriving at hospital in the morning were more likely to receive a brain scan within 1 hour than were those admitted in the afternoon. This suggests that the focus on the weekend has perhaps underestimated the true extent and nature of time-based variations in care. Indeed, the new paper by Anselmi et al found that the excess mortality risk was not uniformly distributed even over the weekend, and peaked for patients who were admitted on Sunday daytimes. ${ }^{7}$ Intriguingly, it might be possible to use pattern recognition to help to move beyond merely observing the weekend effect (and other variations in quality) to identifying likely causes that can be the focus of quality improvement efforts.

\section{WHERE NEXT FOR POLICY AND PRACTICE?}

It is challenging to address a problem that is poorly understood, and which has resisted simple explanations or solutions throughout 15 years of research. But if higher mortality rates at the weekend are not simply a data artefact, then the implications for patient safety warrant careful attention. Beyond monitoring this aspect of care quality, what is a practical way forward for healthcare policy and practice? Answering this question means considering how much effort and resource should be spent in eradicating the weekend effect compared with other improvement priorities. And, if the weekend effect is prioritised, what aspects of the organisation or delivery of healthcare need to be changed?

Recent controversies in the National Health Service (NHS) in England about the weekend effect illustrate the tensions raised by these questions. The provision of 7-day health services was identified as a policy priority for the English NHS in 2012. ${ }^{21}$ Implementation has been led centrally, and hospitals are obliged to meet four priority standards relating to 7-day access to emergency care ${ }^{22}$ : first consultant review within 14 hours of admission; access to diagnostics within 24 hours for non-urgent patients (faster for urgent patients); timely access to consultant-directed interventions and review twice daily for patients receiving high-dependency care (once daily after transfer to a general ward). ${ }^{23}$ Hospitals are expected to achieve $100 \%$ compliance with these indicators by 2020 , and implementation has been carried out using a mixture of contractual arrangements, financial incentives, sharing of case studies and support from regional improvement teams. As these indicators are not collected by existing electronic data returns, hospitals are largely obliged to collect the data through retrospective review of a sample of case records.

The new standards imply that there is an expectation that the focus on the working practices of senior hospital doctors at the weekend (rather than any other clinical staff group) will improve care quality at the weekend and reduce the weekend effect. However, healthcare is a complex adaptive system, and there are many cautionary tales of where increased performance against process metrics did not lead to the anticipated improvements in outcomes. ${ }^{24}$ Part of the challenge is that, often, the root causes of problems differ according to local context, and initiatives can fail without sufficient investment in understanding the nature of the specific problems faced in each locality. ${ }^{25}$ Furthermore, efforts to address systems-level problems like the weekend effect need to think beyond the walls of hospitals. Delivering timely care within hospital settings depends on improving processes within the hospital and on how patients move through the wider healthcare system. ${ }^{26}$ This is partly because the flow of patients within hospitals depends on how people access urgent care, the availability of primary care and whether social care or rehabilitation services are available to support discharge from hospital. For example, there is evidence that extending general practice opening hours can reduce pressures on emergency departments, for minor, patient-initiated attendances. ${ }^{27}$

\section{SUMMARY}

Untangling the evidence for the weekend effect has proven extremely difficult, and it therefore comes as no surprise that it has generated controversy in some settings. Messy, conflicting evidence is, however, less the exception than the norm when it comes to questions about healthcare quality, and meaningful progress can be made even for these knotty types of problems through the use of quality improvement methods. Actively looking for temporal variation in quality of care, patient safety and outcomes should 
help ensure that potentially important, systematic inequalities in quality do not persist unnoticed.

Correction notice This article has been edited since it it first appeared Online First. The opening sentence has been corrected.

Acknowledgements We are grateful for comments received from Tim Gardner, Therese Lloyd, Shaun Leamon and Will Warburton on an earlier draft.

Competing interests None declared.

Provenance and peer review Commissioned; internally peer reviewed.

\section{REFERENCES}

1 Bell CM, Redelmeier DA. Mortality among patients admitted to hospitals on weekends as compared with weekdays. N Engl J Med 2001;345:663-8.

2 Lilford RJ, Chen Y-F. The ubiquitous weekend effect: moving past proving it exists to clarifying what causes it. BMJ Qual Saf $2015 ; 24: 480-2$.

3 Freemantle N, Ray D, McNulty D, et al. Increased mortality associated with weekend hospital admission: a case for expanded seven day services? BMJ 2015;4596:1-6.

4 NHS England. Five year forward view. London: NHS England, 2014. http://www.england.nhs.uk/wp-content/uploads/2014/10/ 5 yfv-web.pdf

5 Goddard AF. Lessons to be learned from the UK junior doctors' strike. JAMA 2016;316:1445-6.

6 McKee M. The weekend effect: now you see it, now you don't. BMJ 2016;353:i2750.

7 Anselmi L, Meacock R, Kristensen SR, et al. Arrival by ambulance explains variation in mortality by time of admission: retrospective study of admissions to hospital following emergency department attendance in England. BMJ Qual Saf 2017; 26:613-21.

8 Mohammed MA, Faisal M, Richardson D, et al. Adjusting for illness severity shows there is no difference in patient mortality at weekends or weekdays for emergency medical admissions. QJM 2016: doi: 10.1093/qjmed/hcw104.

9 Deeny SR, Steventon A. Making sense of the shadows: priorities for creating a learning health care system based on routinely collected data. BMJ Qual Saf 2015;24:505-15.

10 Austin PC, Mamdani MM, Stukel TA, et al. The use of the propensity score for estimating treatment effects: administrative versus clinical data. Stat Med 2005;24:1563-78.

11 Concha OP, Gallego B, Hillman K, et al. Do variations in hospital mortality patterns after weekend admission reflect reduced quality of care or different patient cohorts? A population-based study. BMJ Qual Saf 2014;23:215-22.

12 Li L, Rothwell PM, Oxford Vascular Study. Biases in detection of apparent 'weekend effect' on outcome with administrative coding data: population based study of stroke. BMJ 2016;353: i2648.
13 Meacock R, Anselmi L, Kristensen SR, et al. Higher mortality rates amongst emergency patients admitted to hospital at weekends reflect a lower probability of admission. J Health Serv Res Policy 2016. http://dx.doi.org/10.1177/ 1355819616649630

14 Bray BD, Cloud GC, James MA, et al. Weekly variation in health-care quality by day and time of admission: a nationwide, registry-based, prospective cohort study of acute stroke care. Lancet 2016;388:170-7.

15 Kostis WJ, Demissie K, Marcella SW, et al. Weekend versus weekday admission and mortality from myocardial infarction. N Engl J Med 2007;356:1099-109.

16 Aldridge C, Bion J, Boyal A, et al. Weekend specialist intensity and admission mortality in acute hospital trusts in England: a cross-sectional study. Lancet 2016;6736:1-9.

17 Ruiz M, Bottle A, Aylin PP. Exploring the impact of consultants' experience on hospital mortality by day of the week: a retrospective analysis of hospital episode statistics. BMJ Qual Saf 2016;25:337-44.

18 Bray BD, Ayis S, Campbell J, et al. Associations between stroke mortality and weekend working by stroke specialist physicians and registered nurses: prospective multicentre cohort study. PLoS Med 2014;11:e1001705.

19 Agrawal S, Garg L, Sharma A, et al. Comparison of inhospital mortality and frequency of coronary angiography on weekend versus weekday admissions in patients with non-ST-segment elevation acute myocardial infarction. Am J Cardiol 2016;118:632-4.

20 Turner M, Barber M, Dodds H, et al. Stroke patients admitted within normal working hours are more likely to achieve process standards and to have better outcomes. J Neurol Neurosurg Psychiatr 2016;87:138-43.

21 NHS Commissioning Board. Everyone counts: planning for patients 2013/14. Leeds: NHS Commissioning Board, 2012. $1-36$.

22 NHS England, NHS Improvement. NHS operational planning and contracting guidance 2017-2019. London: NHS England and NHS Improvement, 2016.

23 NHS Improvement. Seven day services clinical standards. London: NHS, 2016.

24 Lee JS, Primack BA, Mor MK, et al. Processes of care and outcomes for community-acquired pneumonia. Am J Med 2011;124:1175.e9-17.

25 Sutton E, Dixon-Woods M, Tarrant C. Ethnographic process evaluation of a quality improvement project to improve transitions of care for older people. BMJ Open 2016;6: e010988.

26 The Health Foundation. Improving patient flow. London: The Health Foundation, 2013. http://www.health.org.uk/sites/ default/files/ImprovingPatientFlow_fullversion.pdf

27 Whittaker W, Anselmi L, Kristensen SR, et al. Associations between extending access to primary care and emergency department visits: a difference-in-differences analysis. PLOS Med 2016;13:e1002113. 